\title{
A cynomolgus monkey model of carotid atherosclerosis induced by puncturing and scratching of the carotid artery combined with a high-fat diet
}

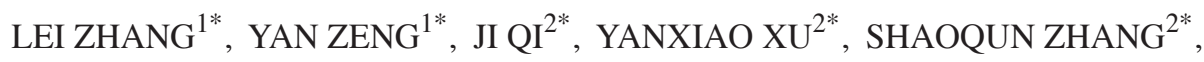 \\ XIN ZHOU ${ }^{2}$, RUIYUE PING ${ }^{3}$ and SHIJIE FU ${ }^{1 *}$ \\ ${ }^{1}$ Orthopedics Department, Affiliated Traditional Chinese Medicine Hospital of Southwest Medical University, Luzhou, \\ Sichuan 646600; ${ }^{2}$ School of Traditional Chinese Medicine, Southern Medical University, Guangzhou, Guangdong 510515; \\ ${ }^{3}$ Department of Dermatology, The Second Affiliated Hospital, Guangzhou University of Traditional Chinese Medicine, \\ Guangzhou, Guangdong 510403, P.R. China
}

Received October 3, 2017; Accepted April 6, 2018

DOI: $10.3892 /$ etm.2018.6143

\begin{abstract}
Cardio-cerebrovascular disease is one of the three major causes of mortality in humans and constitutes a major socioeconomic burden. Carotid atherosclerosis (CAS) is a very common lesion of the arterial walls, which leads to narrowing of the arteries, in some cases occluding them entirely, increasing the risk of cardiovascular events. The aim of the present study was to evaluate a cynomolgus monkey model of carotid atherosclerosis (CAS) induced by puncturing and scratching combined with a high-fat diet. A total of 12 cynomolgus monkeys were randomly divided into four groups: A, puncturing and scratching carotid artery intimas + high-fat diet $(n=3) ; B$, puncturing and scratching carotid artery intimas + regular diet $(n=3)$; C, high-fat diet only $(n=3)$; and $\mathrm{D}$, regular diet only $(\mathrm{n}=3)$. Blood was harvested at weeks 4 , 6 and 8 and plasma lipid levels were assessed. At week 8, monkeys were sacrificed and carotid arteries were harvested for hematoxylin and eosin (H\&E) staining to observe pathological changes. The results revealed that a high-fat diet led to increased plasma lipid levels and accelerated plaque formation. Carotid color Doppler ultrasonography was performed and, along with H\&E staining, revealed plaque formation in group A. In summary, the results of the present study suggest
\end{abstract}

Correspondence to: Professor Shijie Fu, Orthopedics Department, Affiliated Traditional Chinese Medicine Hospital of Southwest Medical University, 182 Chun Hui Road, Luzhou, Sichuan 646600, P.R. China

E-mail: fushijieggj@126.com

*Contributed equally

Abbreviations: CAS, carotid atherosclerosis; LDL-C, low-density lipoprotein cholesterol; TG, triglycerides; TC, total cholesterol; HDL-C, high-density lipoprotein cholesterol

Key words: carotid atherosclerosis, animal models, high-fat diet that a cynomolgus monkey model of CAS model may be successfully constructed by puncturing and scratching of the carotid artery intimas in combination with a high-fat diet.

\section{Introduction}

Cardio-cerebrovascular disease is a major cause of mortality in humans and constitutes a major socioeconomic burden. According to epidemiological surveys, the mean incidence of acute brain cardiovascular stroke (BCS) events is 116/100,000, with a mortality rate of $81 / 100,000$ (1). Ischemic cerebrovascular diseases account for $>70 \%$ of all cerebrovascular diseases and the incidence increases annually (2). In 2005, 5.7 million patients succumbed to BCS, $87 \%$ of whom resided in low-income countries (3). The World Health Organization (WHO) predicts that the number of cardiovascular-associated mortalities will reach 20.5 million annually by 2020 (4).

Carotid atherosclerosis (CAS) is a common lesion of the arterial walls, which leads to artery narrowing and occlusion and is the basis for the development of cardiovascular events (5). CAS mainly comprises the thickening, hardening and restructuring of the arterial walls (6-9). CAS is predominantly observed in great or middle arterial walls and typically presents with a build up of lipid, cells, cellular matrix and calcium salt deposition on the arterial intima, followed by intimal thickening, structural failure, arterial wall deformation and narrowing of the arterial lumen $(10,11)$. A number of mechanisms of CASE have been suggested, including the thrombosis theory, lipid infiltration theory, monoclonal theory, damage reaction hypothesis, oxidative stress hypothesis, stem cell formation hypothesis, immune dysfunction hypothesis, homocysteine and arginine hypothesis and the inflammatory reaction theory (12-14). The damage reaction hypothesis (15-18) suggests that the inflammatory response following vascular endothelial cell damage is an important factor promoting the pathogenesis of atherosclerosis as it is associated with a series of vascular intimal changes, including endothelial cell damage, increased endothelial cell permeability and adhesion, increased blood coagulation and cytokine release and increased growth factors, ultimately resulting in CAS 
development. However, researchers supporting the lipid infiltration theory $(6,19-21)$ reported that the occurrence and progression of CAS are associated with abnormal lipid metabolism, mainly the accumulation of cholesterol, low-density lipoprotein cholesterol (LDL-C) in particular, in endothelial cells.

In summary, vascular intimal damage combined with elevated plasma lipid levels eventually lead to the formation of CAS, followed by vascular stenosis and occlusion. At present, the lack of an optimal and effective CAS model limits our ability to study its pathogenesis and potential treatments; research is therefore required to develop an efficient CAS model that is easy to construct, exhibits the typical pathological changes observed in CAS and is suitable for intervention treatment.

A number of CAS models have been developed, including those utilizing high-fat diets $(20,21)$, mixed diets (22-24), endothelial damage (16-18), inflammation and the immune response (25-27), the hemodynamic method (28-30) and the genetic engineering method (31-33); however, each method comes with its own pros and cons. The high-fat diet method is simple, cost-effective and may induce atherosclerosis in the coronary arteries and brain, but the modeling time is long (3-5 months) and the development of CAS is not guaranteed. It is difficult to form CAS plaques similar to those observed in humans and secondary infections could develop, affecting the stability of the experiment (34-37). This may be associated with the luminal structures of local arteries, hemodynamic characteristics and endothelial cell function. In addition, the balloon injury method combined with the use of a high-fat diets, although it may shorten CAS modeling time, is controversial (38-40), due to the complex surgery, plaque instability and risk of thrombosis; in addition, the balloon size was poorly controlled, which may cause arterial rupture and increase the risk of model failure. With regards to the genetic engineering method, it was demonstrated (41) that, although rabbits with natural defects or genetic modifications by artificial cultivation could develop CAS, constructing a CAS model was high-cost, time-consuming and difficult to apply. The choice of experimental animals is one of the key considerations in CAS modeling. Rats, rabbits and pigs are currently the most commonly used experimental animals for the study of CAS (42-44); however, due to their physiological and structural differences with humans, optimal efficacy often cannot be achieved. Cynomolgus monkeys are primates that are physiologically and structurally similar to humans; furthermore, they are easily fed, sensitive to a high-fat diet and readily develop CAS (45). Therefore, cynomolgus monkeys were selected as the experimental animals in the present study.

Based on the aforementioned theories and objectives, puncturing and scratching of the carotid artery intima combined with a high-fat diet were used in the present study to develop a CAS model in cynomolgus monkeys. This method shortens the time required for modeling and improves the model quality; furthermore, this model may accurately imitate the plaque formation process that occurs in humans under hyperlipidemic conditions.

\section{Materials and methods}

Ethical statement. All procedures were approved by the Ethical Inspection Committee of Animal Experiments of Yunnan
Yinmore Biological Technology Co., Ltd. (no. YBT1602; Xishuangbanna, Yunnan, China).

Animals. A total of 12 male cynomolgus monkeys (age, 4.0-5.0 years; weight, 6.0-7.0 kg) were purpose-bred and purchased from Yunnan Yinmore Biological Technology Co., Ltd., which is an Association for Assessment and Accreditation of Laboratory Animal Care International (Jinghong City, China) accredited animal research facility.

Monkeys were housed at the Laboratory Animal Breeding Center of Yunnan Yinmore Biological Technology Co., Ltd. in stable cages. For sleeping, feeding and rest, cages measured $1.5 \times 2 \times 1.5 \mathrm{~m}$. The housing conditions were as follows: $12 \mathrm{~h}$ light/dark cycle at $22-24^{\circ} \mathrm{C}$ with a relative humidity of $45-65 \%$. Water was available ad libitum via water bottles. Monkeys were moved out of cages to an activity room measuring $4 \times 12.5 \times 8 \mathrm{~m}$ for $6-8 \mathrm{~h}$ per day.

Monkeys were randomly divided into four groups $(n=3)$ : A, puncturing and scratching of carotid artery intimas + high-fat diet (500 g/day); B, puncturing and scratching of carotid artery intimas + with a regular diet (500 g/day); C, high-fat diet (500 g/day); and D, blank control with a regular diet (500 g/day) $(\mathrm{n}=3)$. Prior to the experiment, monkeys were adaptively fed for 1 month; individuals in groups $\mathrm{A}$ and $\mathrm{C}$ were fed with a regular diet (Yunnan yinmore Biotechnology Co., Ltd.), containing $100 \%$ ordinary particles feed), monkeys in groups B and D were fed with a high-fat diet (Yunnan yinmore Biotechnology Co., Ltd.), containing 2\% cholesterol, $10 \%$ lard and $88 \%$ ordinary particles feed).

Animal modeling. In groups A and B, the CAS model was constructed in the left carotid artery by puncturing and scratching the carotid artery intima. Monkeys were denied access to food and water prior to surgery and were anesthetized using $5 \mathrm{mg} / \mathrm{kg}$ Zoletil 50 (Virbac, Carros, France). Monkeys were fixed in a dorsal position, the neck was shaved and the skin was disinfected. A 4-cm longitudinal incision was made at $1 \mathrm{~cm}$ laterally from the laryngeal prominence, followed by stepwise separation of the skin and subcutaneous tissues. In the space between the sternocleidomastoid muscle and the laryngeal prominence, the common carotid artery was isolated and fixed with a surgical suture. A 5-ml syringe needle (30 $\mathrm{mm}$ long, $0.6 \mathrm{~mm}$ diameter) was used to pierce the artery and repeatedly scratch the arterial walls, taking caution not to perforate the artery (Fig. 1). The needle was retracted and gauze was applied to the puncture point, following which the wound was washed and sutured.

All surgeries were completed by the same group of surgeons. On postoperative day 3 , levofloxacin hydrochloride and sodium chloride injection (Jiangsu hausen Pharmaceutical Group Co., Ltd., Jiangsu, China; $8 \mathrm{mg} / \mathrm{kg}$ twice daily intravenously) were administered to prevent infection and the wound was closely observed; tramadol hydrochloride (Shijiazhuang Pharmaceutical Group Co., Ltd, Hebei, China; $2 \mathrm{mg} / \mathrm{kg}$, once daily intramuscularly) was administered for pain relief.

Following surgery, monkeys in group A were fed a high-fat diet (containing 2\% cholesterol, 10\% lard and 88\% regular granulated feed), whereas the monkeys in group B were fed a regular diet for 8 weeks. During this period, the wound condition, eating and swallowing were observed. Furthermore, blood 


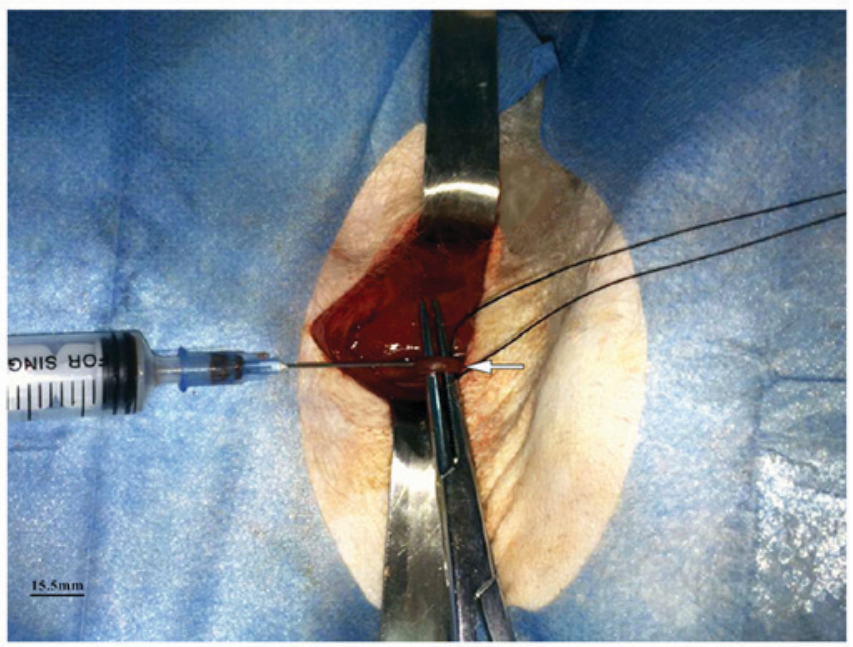

Figure 1. The cynomolgus monkey model of carotid atherosclerosis was constructed by puncturing the carotid artery and scratching the intima. The white arrow indicates the point at which the carotid artery was pierced with a needle.

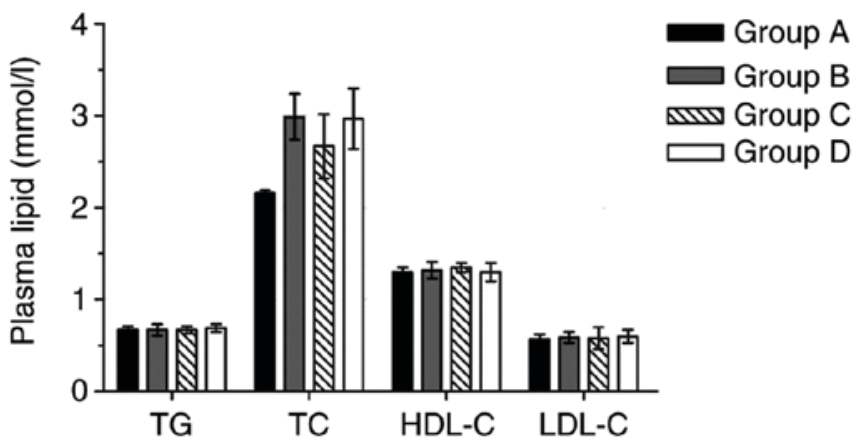

Figure 2. Plasma lipid levels in each group prior to intervention. No statistically significant differences were observed. TG, triglycerides; TC, total cholesterol; HDL-C, high-density lipoprotein cholesterol; LDL-C, low-density lipoprotein cholesterol.

was harvested and plasma lipid levels were measured at weeks 4, 6 and 8 postoperatively. Color Doppler ultrasonography was performed at week 8 monkeys were euthanized. The carotid arteries were harvested to perform hematoxylin and eosin (H\&E) staining and evaluate the pathological changes.

Examination of plasma lipid levels. Plasma lipid levels were measured in all groups prior to surgery and at weeks 4, 6 and 8 postoperatively. A total of 2-3 $\mathrm{ml}$ fasting venous blood was collected, the serum was separated by centrifugation (radius, $18 \mathrm{~cm}$ ) at a speed of $1,814.4 \mathrm{x} \mathrm{g}$ for $5 \mathrm{~min}$ at room temperature and stored at $-80^{\circ} \mathrm{C}$. Plasma lipid levels were measured using an automatic biochemical analyzer (Selectra-E; ELITechGroup, Paris, France). The measured indicators included triglycerides (TG), total cholesterol (TC), low-density lipoprotein cholesterol (LDL-C) and high-density lipoprotein cholesterol (HDL-C).

Carotid color Doppler ultrasonography examination. At week 8 , all monkeys were anesthetized with $5 \mathrm{mg} / \mathrm{kg}$ Zoletil and placed in a supine position with their arms at the sides and the neck was fully exposed. CAS was assessed using a color Doppler ultrasonic diagnostic instrument (Philips Medical Systems B.V., Eindhoven, The Netherlands). The scan evaluated the characteristics of the CAS plaques (low-, mixed- and high-echo plaques) and the rate of vascular stenosis (cross-sectional area of the plaque/cross-sectional area of the whole vessel x100) (46).

According to the standard of the Radiological Society of North America in 2003 (47), carotid stenosis can be classified as mild (rate of vascular stenosis: $<50 \%$ ), moderate (rate: 50-69\%), severe (rate: 70-99\%) or total occlusion (rate: 100\%).

Histology. Following carotid color Doppler ultrasonography examination all monkeys were sacrificed. Part of the carotid artery was harvested, rinsed with isotonic saline, fixed at room temperature for $24 \mathrm{~h}$ in $4 \%$ paraformaldehyde and dehydrated in a graded series of alcohol. Tissues were embedded in paraffin and $5 \mu \mathrm{m}$ sections were prepared using a rotary microtome (Leica Microsystems GmbH, Wetzlar, Germany). H\&E staining was performed on tissue sections at room temperature for $3 \mathrm{~min}$. The typical atherosclerotic histological changes were examined using an optical microscope (magnification, $\mathrm{x} 8$ ).

Statistical analysis. All data are presented as the mean \pm standard error. In order to determine whether the data were normally distributed, the Kolmogorov-Smirnov and Shapiro-Wilk tests were conducted. A repeated measures analysis of variance was performed with Fisher's post hoc test. Statistical analysis was using SPSS version 20.0 (IBM Corp., Armonk, NY, USA) and $\mathrm{P}<0.05$ was considered to indicate a statistically significant difference.

\section{Results}

Changes in plasma lipid levels. Prior to intervention, all plasma lipid levels were within the normal range (Normal ranges: TC, 5.23-5.69 M/l; TG, 0.56-1.7 M/l; HDL-C, >1 mmol/l; LDL-C, $<3.12 \mathrm{mmol} / \mathrm{l}$.) and no statistically significant differences were observed between the groups (Fig. 2). However, plasma TG was significantly increased at weeks 6 and 8 compared with week 4 in groups $\mathrm{A}$ and $\mathrm{C}(\mathrm{P}<0.05$; Fig. 3A). In groups $\mathrm{A}$ and $\mathrm{C}$, TC and HDL-C levels were significantly increased at weeks 6 and 8 compared with week $4(\mathrm{P}<0.05$; Fig. $3 \mathrm{~B}$ and $\mathrm{C})$. However, no significant differences in TC and HDL-C were observed between weeks 6 and 8 in groups $\mathrm{A}$ and $\mathrm{C}$. No significant differences in TG, TC or HDL-C were observed between any time points in groups B and D (Fig. 3A-C). TG, TC and HDL-C levels in groups $\mathrm{A}$ and $\mathrm{C}$ were significantly increased compared with group $\mathrm{D}$ at all time points $(\mathrm{P}<0.05$; Fig. 3A-C). However, no significant difference in TG, TC and HDL-C were observed between groups $\mathrm{A}$ and $\mathrm{C}$ or between groups B and D at any time point (Fig. 3A-C). No significant differences in LDL-C levels were observed between groups or within groups at different time points (Fig. 3D).

Carotid color Doppler ultrasonography. In group A, color Doppler ultrasound revealed mild stenosis (rate: 5-7\%) and high-echo plaques, which were irregular with a rough surface, with discontinuous linear high-echo plaques (fibrous cap) on the arterial walls (Fig. 4). No plaque formation was observed in groups B, C or D. 

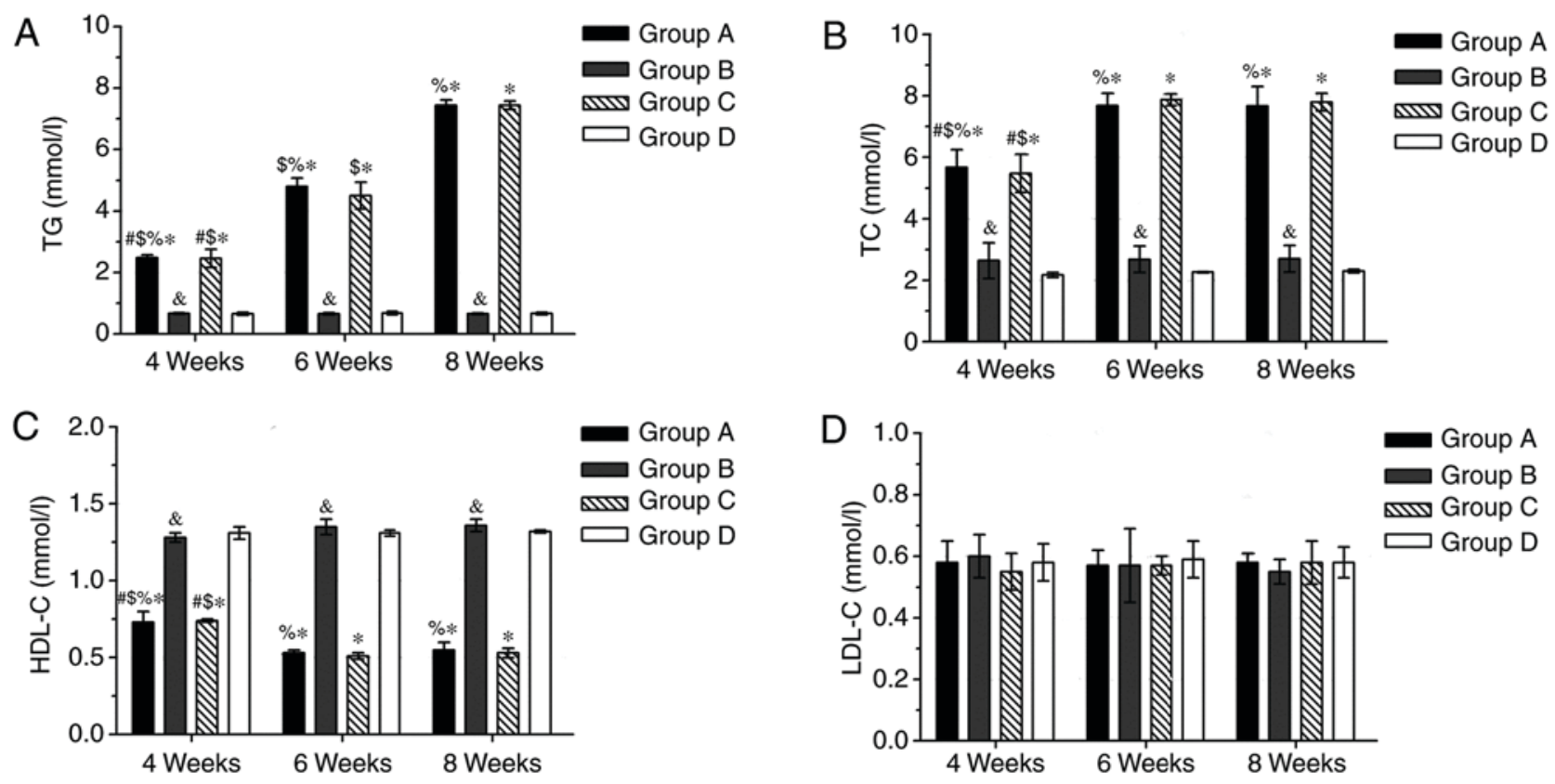

Figure 3. Plasma (A) TG, (B) TC, (C) HDL-C and (D) LDL-C levels in each group at different time points. " $\mathrm{P}<0.05$ vs. 6 weeks in the same group; ${ }^{\$} \mathrm{P}<0.05$ vs. 8 weeks in the same group; ${ }^{\%} \mathrm{P}<0.05$ vs. group B at the same time; ${ }^{\circ} \mathrm{P}<0.05$ vs. group $\mathrm{C}$ at the same time; ${ }^{\prime} \mathrm{P}<0.05$ vs. group $\mathrm{D}$ at the same time. TG, triglycerides; TC, total cholesterol; HDL-C, high-density lipoprotein cholesterol; LDL-C, low-density lipoprotein cholesterol.
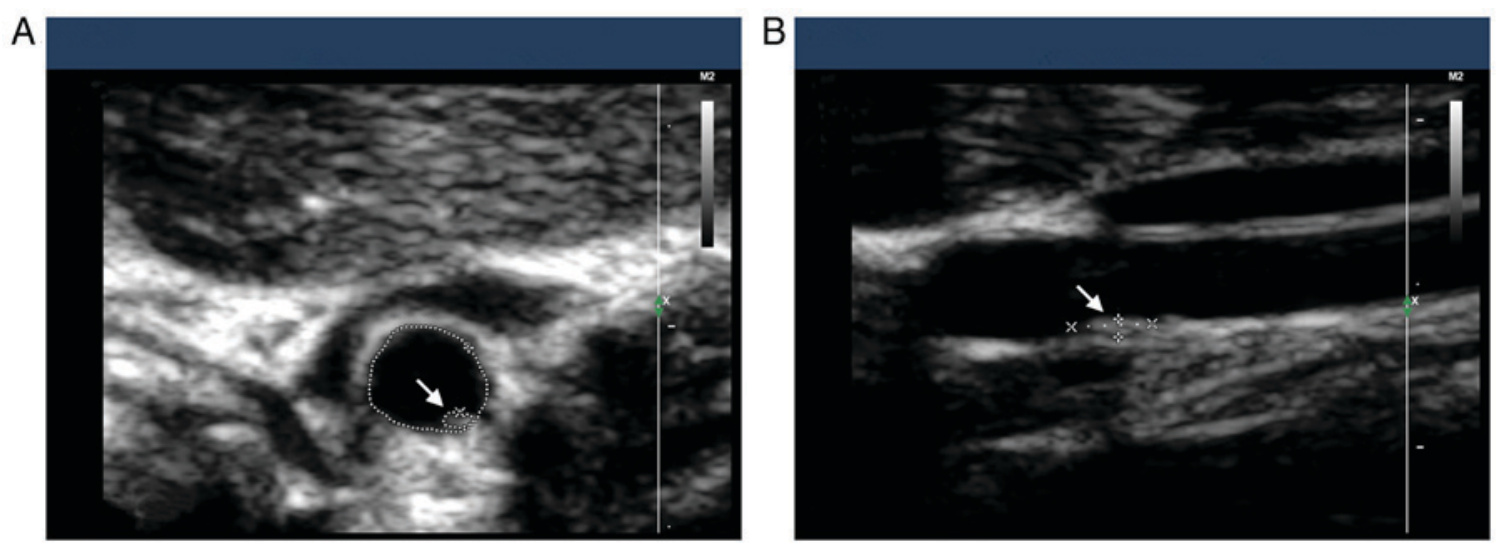

Figure 4. Color Doppler ultrasound of the carotid artery. Images of (A) an artery transect and (B) a longitudinal section were obtained. White arrows indicate plaque formation.

Histological observations. In groups A and B, the intima was obviously injured and thickened, with sloughing off of the endothelial cells (Fig. 5A-D). However, in group A, the plaques on the vessel wall were covered with fibrous tissue, with obvious hyperplasia of the lipid foam cells underneath the intima (Fig. 5A and B). In groups C and D, the arterial wall was complete and the intima was composed of a monolayer of endothelial cells adherent to the elastic plate (Fig. 5E-H). The endothelial cell layer maintained its integrity and the diameter of the lumen was uniform.

\section{Discussion}

There are certain associations between CAS plaque formation, brain cardiovascular stroke and coronary heart disease. The basic pathological changes observed in CAS include intimal deposition of lipids, intimal focal fibrosis and plaque formation, which result in hardening of the vessel wall, luminal stenosis and ischemic changes in the corresponding organs $(6,10,48,49)$. The risk factors of CAS mainly include hyperlipidemia, hypertension, high blood glucose (diabetes), smoking and age (50). CAS may be caused by a variety of factors, including thrombosis, lipid infiltration, injury response, oxidative stress, the stem cell formation theory, immune dysfunction, the homocysteine and arginine hypothesis and the inflammatory reaction theory $(12-14,51)$. However, due to limitations associated with medical technology, social and environmental complexity, researchers have yet to fully elucidate the pathogenesis of CAS $(52,53)$. Therefore, a highly efficient optimal modeling method of CAS is crucial for studying the pathogenesis and treatment of cardio-cerebrovascular disease. The model must be easy to construct, must mimic the formation of CAS plaques in humans under hyperlipidemic conditions and must be suitable for interventional treatment. 

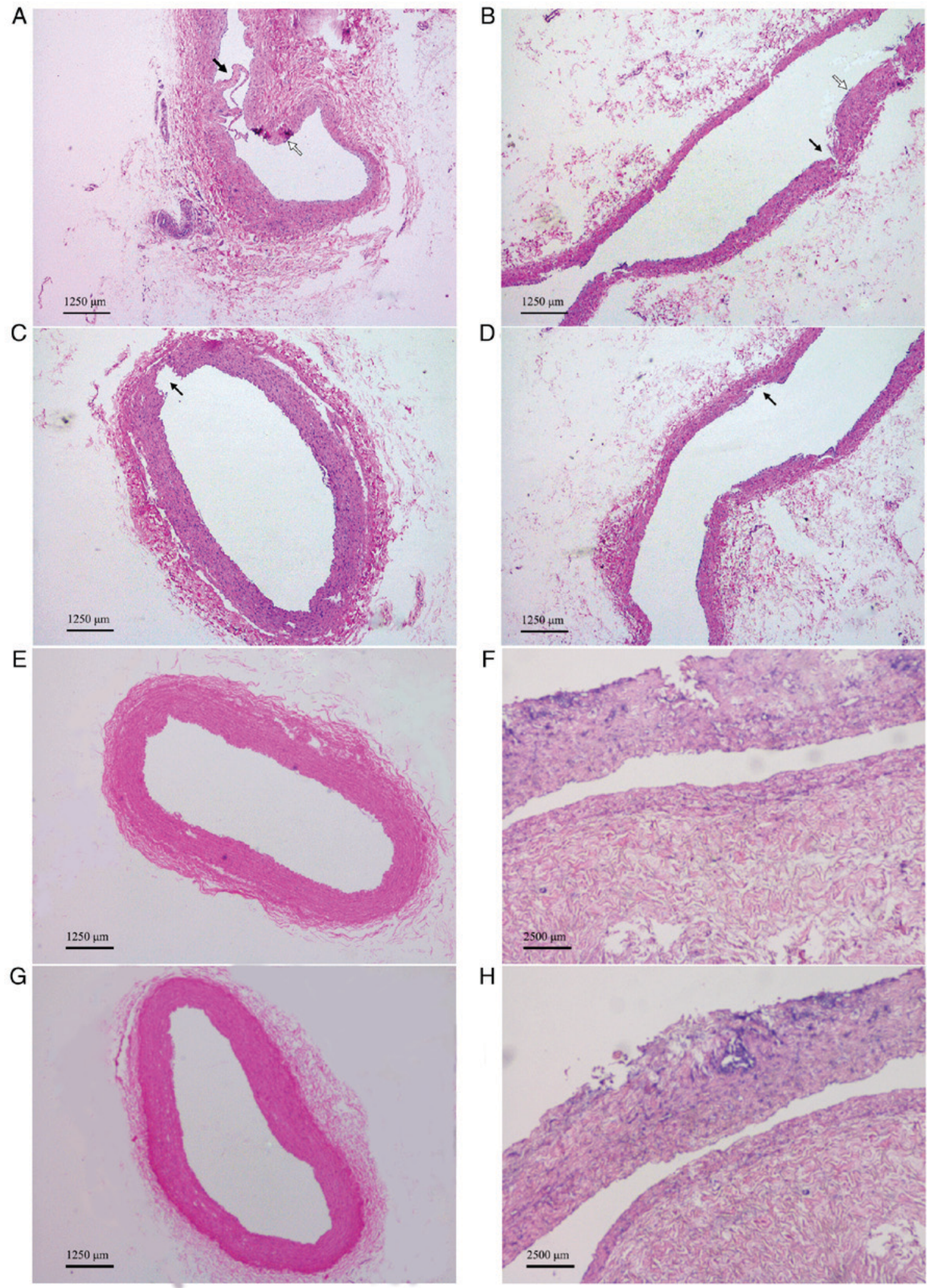

Figure 5. Hematoxylin and eosin staining revealed the morphological characteristics of carotid atherosclerosis. (A) Transect and (B) longitudinal section of the carotid artery in group A. (C) Transect and (D) longitudinal section of the carotid artery in group B. (E) Transect and (F) longitudinal section of the carotid artery in group C. (G) Transect and (H) longitudinal section of the carotid artery in group D. Black arrows indicate scratch injuries on the carotid intima. White arrows indicate the location of atherosclerotic plaques. In groups $\mathrm{C}$ and $\mathrm{D}$, the arterial wall was complete, the endothelial cell layer maintained its integrity and the diameter of the lumen was uniform.

In the present study, on the basis of carotid artery intimal injury, changes in plasma lipids were associated with CAS. Lipid TG levels in groups A and C increased over time, indicating that TG may be used as a specific indicator of plaque formation and the development of CAS. TC and HDL-C increased at week 4 and remained stable at weeks 6 and 8, which suggests 
that TC and HDL-C increases occur during the early stages of CAS development. However, LDL-C levels were not associated with CAS formation in the present study. TG, TC and HDL-C levels were greater in groups $\mathrm{A}$ and $\mathrm{C}$ compared with group $\mathrm{D}$; furthermore, no significant changes in plasma lipid levels were observed in groups B and D over time. These results suggest that a high-fat diet may promote increases in plasma lipid levels and accelerate plaque formation.

In the present study, the carotid artery intima was injured by puncturing and scratching with a needle, which results in platelets and leukocytes adhering to the intima and intimal hyperplasia (54). When intimal injury was coupled with a high-fat diet, CAS plaque formation was observed; however, puncturing and scratching the intima alone, or a high-fat diet alone, did not result in the formation of CAS plaques. In addition, the pathological changes observed during the development of CAS were similar to those reported in humans, including early degeneration of endothelial cells, deposition of foam cells, endothelial proliferation, gradual thickening of the endothelium, thinning of the membrane, lipid deposition, fibrous plaques and the formation of atheromatous plaques $(55,56)$. As such, the cynomolgus monkey model of CAS induced by puncturing and scratching combined with a high-fat diet was deemed to be an optimal, highly efficient, simple and feasible modeling method.

Several details are crucial for the success of the model: i) The modeling method must include intimal injury and a high-fat diet to accelerate the formation of CAS; ii) scratching of the artery wall must be performed with caution to avoid perforating the artery; iii) differences in animal weight should be kept to a minimum; iv) deep anesthesia should be avoided and local anesthesia used instead where possible; v) separation of the skin and subcutaneous connective tissues must be performed with caution to avoid damaging vessels and peripheral nerves, while fully exposing the carotid artery; vi) needle retraction must be followed by gauze oppression hemostasis to prevent arterial hemorrhage; and vii) following surgery, the condition of the wound, swallowing and eating must be monitored.

The modeling method used in the present study has several advantages. Firstly, the procedure is simple and has a low animal mortality rate. Secondly, the duration required for CAS formation is short and the model is easily constructed, as well as stable and reliable. Thirdly, it is possible to assess whether CAS formation is associated with changes in plasma lipid levels. Using color Doppler ultrasound to visualize CAS allows for accurate assessment of stenosis and plaque. Finally, the pathological changes associated with CAS are similar in monkeys and humans; as such, this model may be suitable for studying the pathogenesis of CAS and potential drug interventions. Together, these advantages make this model suitable for studying the prevention and control of CAS and may be more widely applied as a novel modeling method.

However, the present study has some limitations. Firstly, the sample size was relatively small. Due to the cost of obtaining cynomolgus monkeys and funding limitations, only 12 experimental animals were used as research subjects in the present study. However, the sample size was sufficient for basic requirements. Secondly, only H\&E staining was performed for histopathology and the histological and Doppler analyses were not repeated. Meanwhile, high magnification images were not captured. Thirdly, arteries should have been perfusion fixed in order to better assess lumen area changes. Finally, due to the scratch-method used there was some variation in injury and the subsequent degree of plaque formation between individuals. To address the abovementioned limitations, the authors aim to gradually improve the scientific nature of this model for future studies.

\section{Acknowledgements}

The authors would like to thank the Laboratory Animals Breeding Center of Yunnan Yinmore Biological Technology Co., Ltd. (Yunnan, China)

\section{Funding}

The present study was supported by the Science and Research Project of Education Department of Sichuan Province (grant no. 17ZB0472) and the Science and Technology Project of Office of Science and Technology of Luzhou (grant no. 2016-176-13).

\section{Availability of data and materials}

All data generated or analyzed during this study are included in this published article.

\section{Authors' contributions}

LZ and SF designed the study and wrote the manuscript; YZ, JQ and YX performed the experiments and obtained data; SZ analyzed and interpreted the data and finalized the manuscript; XZ and RP assisted in performing the experiments and analysing the data, processed the images and proofread the manuscript.

\section{Ethics approval and consent to participate}

All procedures were approved by the Ethical Inspection Committee of Animal Experiments of Yunnan Yinmore Biological Technology Co., Ltd. (no. YBT1602).

\section{Consent for publication}

Not applicable.

\section{Competing interests}

All authors declare that they have no competing interests.

\section{References}

1. Belcaro G, Nicolaides AN, Ramaswami G, Cesarone MR, De Sanctis M, Incandela L, Ferrari P, Geroulakos G, Barsotti A, Griffin M, et al: Carotid and femoral ultrasound morphology screening and cardiovascular events in low risk subjects: A 10-year follow-up study (the CAFES-CAVE study(1). Atherosclerosis 156: 379-387, 2001.

2. Zhao D, Liu J, Wang W, Zeng Z, Cheng J, Liu J, Sun J and Wu Z: Epidemiological transition of stroke in China: Twenty-one-year observational study from the Sino-MONICA-Beijing project. Stroke 39: 1668-1674, 2008. 
3. Strong K, Mathers C and Bonita R: Preventing stroke: Saving lives around the world. Lancet Neurol 6: 182-187, 2007.

4. Kadota K, Takamura N, Aoyagi K, Yamasaki H, Usa T, Nakazato M, Maeda T, Wada M, Nakashima K, Abe K, et al: Availability of cardio-ankle vascular index (CAVI) as a screening tool for atherosclerosis. Circ J 72: 304-308, 2008.

5. Hong H, Wang MS, Liu Q, Shi JC, Ren HM and Xu ZM: Angiographically evident atherosclerotic stenosis associated with myocardial bridging and risk factors for the artery stenosis located proximally to myocardial bridging. Anadolu Kardiyol Derg 14: 40-47, 2014

6. Buja LM: Nikolai N. Anitschkow and the lipid hypothesis of atherosclerosis. Cardiovasc Pathol 23: 183-184, 2014.

7. Ma H, Lin H, Hu Y, Li X, He W, Jin X, Gao J, Zhao N, Pan B and Gao X: Relationship between non-high-density lipoprotein cholesterol and carotid atherosclerosis in normotensive and euglycemic Chinese middle-aged and elderly adults. Lipids Health Dis 16: 55, 2017.

8. Kulyk C, Farina F, Palmieri A, Viaro F, Causin F, Laverda AM and Baracchini C: 'Phacing' a new cause of carotid artery dissection. Neurologist 22: 54-56, 2017.

9. Haemmig S and Feinberg MW: MicroRNAs as harbingers of high-risk carotid artery atherosclerotic disease? Circ Res 120 596-598, 2017.

10. Willeit J and Kiechl S: Biology of arterial atheroma. Cerebrovasc Dis 10 (Suppl 5): S1-S8, 2000.

11. Mitevska IP, Baneva N, Bosevski M and Kostovska ES Prevalence of risk factors and asymptomatic carotid atherosclerosis in diabetic patients screened for silent myocardial ischemia by SPECT myocardial imaging. Nucl Med Rev Cent East Eur 20 3-9, 2017.

12. Chen $\mathrm{Z}$ and $\mathrm{Xu} \mathrm{H}$ : Anti-inflammatory and immunomodulatory mechanism of tanshinone iia for atherosclerosis. Evid Based Complement Alternat Med 2014: 267976, 2014.

13. Hopkins PN: Molecular biology of atherosclerosis. Physiol Rev 93: 1317-1542, 2013.

14. Calvayrac O, Rodríguez-Calvo R, Martí-Pamies I, Alonso J, Ferrán B, Aguiló S, Crespo J, Rodríguez-Sinovas A, Rodríguez C and Martínez-González J: NOR-1 modulates the inflammatory response of vascular smooth muscle cells by preventing $\mathrm{NF \kappa B}$ activation. J Mol Cell Cardiol 80: 34-44, 2015.

15. Lin YW, Liu PS, Adhikari N, Hall JL and Wei LN: RIP140 contributes to foam cell formation and atherosclerosis by regulating cholesterol homeostasis in macrophages. J Mol Cell Cardiol 79: 287-294, 2015.

16. Koniari I, Apostolakis E, Diamantopoulos A, Papadaki H, Papadimitriou E, Poimenidi E, Karnabatidis D, Karahaliou A, Costaridou L, Papalois A, et al: Transauricular balloon angioplasty in rabbit thoracic aorta: A novel model of experimental restenosis. Lipids Health Dis 13: 33, 2014

17. Mehrad H, Mokhtari-Dizaji M, Ghanaati H, Shahbazfar AA and Mohsenifar A: Developing a rabbit model of neointimal stenosis and atherosclerotic fibrous plaque rupture. J Tehran Heart Cent 6 : $117-125,2011$.

18. Nam D, Ni CW, Rezvan A, Suo J, Budzyn K, Llanos A, Harrison D, Giddens D and Jo H: Partial carotid ligation is a model of acutely induced disturbed flow, leading to rapid endothelial dysfunction and atherosclerosis. Am J Physiol Heart Circ Physiol 297: H1535-H1543, 2009.

19. Xu YX, Ashline D, Liu L, Tassa C, Shaw SY, Ravid K, Layne MD, Reinhold V and Robbins PW: The glycosylation-dependent interaction of perlecan core protein with LDL: Implications for atherosclerosis. J Lipid Res 56: 266-276, 2014.

20. Salisbury D and Bronas U: Inflammation and immune system contribution to the etiology of atherosclerosis: Mechanisms and methods of assessment. Nurs Res 63: 375-385, 2014.

21. Niimi M, Keyamura Y, Nozako M, Koyama T, Kohashi M, Yasufuku R, Yoshikawa T and Fan J: Probucol inhibits the initiation of atherosclerosis in cholesterol-fed rabbits. Lipids Health Dis 12: 166, 2013.

22. Chen M: Effects of Chinese herbal compound "Xuemai Ning" on rabbit atherosclerosis model and expression of ABCA1. Int J Biomed Sci 9: 153-161, 2013

23. Julve J, Escolà-Gil JC, Rodríguez-Millán E, Martín-Campos JM, Jauhiainen M, Quesada H, Rentería-Obregón IM, Osada J, Sánchez-Quesada JL and Blanco-Vaca F: Methionine-induced hyperhomo- cysteinemia impairs the antioxidant ability of high-density lipoproteins without reducing in vivo macrophage-specific reverse cholesterol transport. Mol Nutr Food Res 57: 1814-1824, 2013.
24. Guo HY, Xu FK, Lv HT, Liu LB, Ji Z, Zhai XY, Tang WL and Chi JF: Hyperhomocysteinemia independently causes and promotes atherosclerosis in LDL receptor-deficient mice. J Geriatr Cardiol 11: 74-78, 2014.

25. Jia R, Kurita-Ochiai T, Oguchi S and Yamamoto M: Periodontal pathogen accelerates lipid pero-xidation and atherosclerosis. J Dent Res 92: 247-252, 2013

26. Lee WR, Kim KH, An HJ, Park YY, Kim KS, Lee CK, Min BK and Park KK: Effects of chimeric decoy oligodeoxynucleotide in the regulation of transcription factors NF-kB and Spl in an animal model of atherosclerosis. Basic Clin Pharmacol Toxicol 112: 236-243, 2013

27. Deniset JF and Pierce GN: Possibilities for therapeutic interventions in disrupting Chlamydophila pneumonia involvement in atherosclerosis. Fundam Clin Pharmacol 24: 607-617, 2010.

28. Korshunov VA and Berk BC: Flow-induced vascular remodeling in the mouse: A model for carotid intima-media thickening. Arterioscler Thromb Vasc Biol 23: 2185-2191, 2003.

29. Bit A, Ghagare D, Rizvanov AA and Chattopadhyay H: Assessment of influences of stenoses in right carotid artery on left carotid artery using wall stress marker. Biomed Res Int 2017: 2935195, 2017.

30. Zierler RE, Leotta DF, Sansom K, Aliseda A, Anderson MD and Sheehan FH: Development of a duplex ultrasound simulator and preliminary validation of velocity measurements in carotid artery models. Vasc Endovascular Surg 50: 309-316, 2016.

31. Getz GS and Reardon CA: Animal models of atherosclerosis. Arterioscler Thromb Vasc Boil 32: 1104-1115, 2012.

32. Merino H, Parthasarthy S and Singla DK: Partial ligation-induced carotid artery occlusion induces leukocyte recruitment and lipid accumulation-a shear stress model of atherosclerosis. Mol Cell Biochem 372: 267-273, 2013.

33. Du L, Zhang J, De Meyer GR, Flynn R and Dichek DA: Improved animal models for testing gene therapy for atherosclerosis. Hum Gene Ther Methods 25: 106-114, 2014.

34. Nachtigal P, Semecky V, Kopecky M, Gojova A, Solichova D, Zdansky P and Zadak Z: Application of stereological methods for the quantification of VCAM-1 and ICAM-1 expression in early stages of rabbit atherogenesis. Pathol Res Pract 200: 219-229, 2004.

35. Fotis L, Agrogiannis G, Vlachos IS, Pantopoulou A, Margoni A, Kostaki M, Verikokos C, Tzivras D, Mikhailidis DP and Perrea D: Intercellular adhesion molecule (ICAM)-1 and vascular cell adhesion molecule (VCAM)-1 at the early stages of atherosclerosis in a rat model. In Vivo 26: 243-250, 2012.

36. Shen J, Stevenson J, Geng X, Yang J, Yin C, Li F, Wang S, Du H, Ji X and Ding Y: A new clinically relevant model for intracranial atherosclerosis in rats. Neurol Res 38: 817-822, 2016.

37. Palekar RU, Jallouk AP, Myerson JW, Pan H and Wickline SA: Inhibition of thrombin with PPACK-nanoparticles restores disrupted endothelial barriers and attenuates thrombotic risk in experimental atherosclerosis. Arterioscler Thromb Vasc Biol 36: 446-455, 2016.

38. McMahon AC, Kritharides L and Lowe HC: Animal models of atherosclerosis progression: Current concepts. Curr Drug Targets Cardiovasc Haematol Disord 5: 433-440, 2005.

39. Mishra V, Sinha SK and Rajavashisth TB: Role of macrophage colony-stimulating factor in the development of neointimal thickening following arterial injury. Cardiovasc Pathol 25: 284-292, 2016

40. Helkin A, Desai P, Bailey I, Bruch D, Maier KG and Gahtan V: Rat strain determines statin effect on intimal hyperplasia after carotid balloon injury. J Vasc Surg 63: 566-567, 2016.

41. Mercer J and Bennett M: The role of p53 in atherosclerosis. Cell Cycle 5: 1907-1909, 2006

42. Wan SY, Hu YC, Zhan YQ, Qin DD and Ding Y: Hemocoagulase atrox reduces vascular modeling in rabbit carotid artery adventitia. Int J Clin Exp Pathol 6: 2386-2395, 2013.

43. Cui Y, Tian Y, Tang Y, Jia L, Wu A, Peng P, Yang J, Du H, Wang $X$ and $\mathrm{Wu} \mathrm{L}$ : Application of sodium alginate microspheres in ischemic stroke modeling in miniature pigs. Neural Regen Res 8: 1473-1480, 2013.

44. Kurkin DV, Morkovin EI, Verkholiak DV, Osadchenko NA, Tyurenkov IN and Petrov VI: Behavioral phenotyping in rats during the experimental modeling of chronic cerebral circulation disorder. Zh Nevrol Psikhiatr Im S.S. Korsakova 117: 69-73, 2017 (In Russian; Abstract available in Russian from the publisher).

45. Mubiru JN, Garcia-Forey $M$, Higgins PB, Hemmat $P$, Cavazos NE, Dick EJ Jr, Owston MA, Bauer CA, Shade RE, Comuzzie AG and Rogers J: A preliminary report on the feeding of cynomolgus monkeys (Macaca fascicularis) with a high-sugar high-fat diet for 33 weeks. J Med Primatol 40: 335-341, 2011. 
46. Mirza W, Arain M, Ali A, Bari V, Kazim M, Fatima K, Sajjad Z, Jiwani A and Ahmad N: Carotid intima media thickness evaluation by ultrasound comparison amongst healthy, diabetic and hypertensive Pakistani patients. J Pak Med Assoc 66: 1396-1400, 2016.

47. Grant EG, Benson CB, Moneta GL, Alexandrov AV, Baker JD, Bluth EI, Carroll BA, Eliasziw M, Gocke J, Hertzberg BS, et al: Carotid artery stenosis: Grayscale and doppler ultrasound diagnosis-society of radiologists in ultrasound consensus conference. Ultrasound Q 19: 190-198, 2003.

48. Patel AK, Suri HS, Singh J, Kumar D, Shafique S, Nicolaides A, Jain SK, Saba L, Gupta A, Laird JR, et al: A Review on atherosclerotic biology, wall stiffness, physics of elasticity, and its ultrasound-based measurement. Curr Atheroscler Rep 18: 83, 2016.

49. Li P, Pan GP, Jia M, Wang QQ, Guo ZG, Zhao FR, Lei GL, Wan GR and Wan GM: Effect of Xin Mai Jia on atherosclerosis in rats. Genet Mol Res 14: 6018-6027, 2015.

50. Wang X, Li W, Song F, Wang L, Fu Q, Cao S, Gan Y, Zhang W, Yue W, Yan F, et al: Carotid Atherosclerosis Detected by Ultrasonography: A National Cross-Sectional Study. J Am Heart Assoc 7: e008701 2018.

51. Qin G, Chen Z, Su W, Geng X, Chen X, Xu X and Pan W: Clinical usefulness of metabolic risk factors to identify young asymptomatic women adults with subclinical atherosclerosis: A cross-sectional study. Medicine (Baltimore) 96: e6237, 2017.

52. Sun J, Zhao XQ, Balu N, Neradilek MB, Isquith DA, Yamada K Cantón G, Crouse JR III, Anderson TJ, Huston J III, et al: Carotid plaque lipid content and fibrous cap status predict systemic CV outcomes: The mri substudy in AIM-HIGH. JACC Cardiovasc Imaging 10: 241-249, 2017.
53. Hinojosa CA, Anaya-Ayala JE, Laparra-Escareno $\mathrm{H}$, Torres-Machorro A, Lizola R and Gamboa-Domínguez A: Concomitant surgical treatment of symptomatic carotid artery disease with a coexisting shamblin I carotid body tumor. Vasc Endovascular Surg 51: 103-107, 2017.

54. Hirosumi J, Nomoto A, Ohkubo Y, Sekiguchi C, Mutoh S, Yamaguchi I and Aoki H: Inflammatory responses in cuff-induced atherosclerosis in rabbits. Atherosclerosis 64: 243-254, 1987.

55. Xiong Y, Ren YF, Xu J, Yang DY, He XH, Luo JY, Rana JS, Zhang Y, Zheng ZS, Liu DH and Wu GF: Enhanced external counterpulsation inhibits endothelial apoptosis via modulation of BIRC2 and Apaf-1 genes in porcine hypercholesterolemia. Int J Cardiol 171: 161-168, 2014.

56. Van Vré EA, Bosmans JM, Van Brussel I, Maris M, De Meyer GR, Van Schil PE, Vrints CJ and Bult H: Immunohistochemical characterisation of dendritic cells in human atherosclerotic lesions: possible pitfalls. Pathology 43: 239-247, 2011.

This work is licensed under a Creative Commons Attribution-NonCommercial-NoDerivatives 4.0 International (CC BY-NC-ND 4.0) License. 